\title{
The Behavior and Transport of Hydrogen in Silicon Solar Cells Observed through Changes in Contact Resistance
}

\author{
Phillip Hamer ${ }^{1,2}$, Hantao $\mathrm{Li}^{1}$, Catherine Chan ${ }^{2}$, Chandany Sen ${ }^{2}$, Ruy S. Bonilla ${ }^{1}$, Peter Wilshaw ${ }^{1}$ \\ ${ }^{1}$ Department of Materials, The University of Oxford, 16 Parks Rd, Oxford, OX1 3PH, UK \\ ${ }^{2}$ School of Photovoltaic and Renewable Energy Engineering, The University of New South Wales, NSW, \\ 2052, Australia
}

\begin{abstract}
This paper presents a simple method of studying the transport of hydrogen in crystalline silicon, as well as its release from bound forms during annealing at temperatures between $350-450^{\circ} \mathrm{C}$. This technique is based upon observations of an increase in the contact resistance of industrial solar cells, which displays the reversibility and dependence upon electric field commonly associated with hydrogen. It is shown that the increase in contact resistance depends strongly upon whether the device is fabricated on multi- or mono-crystalline silicon and upon the device structure.
\end{abstract}

Index terms - hydrogen, crystalline silicon, screen-printed contacts, photovoltaics

\section{INTRODUCTION}

The observation of hydrogen in crystalline silicon solar cells is one of the most frustrating challenges in the field. Hydrogen is capable of radically altering the behavior of solar cells while remaining well below the detection limit of available characterization techniques. Hydrogen has traditionally been viewed as beneficial to solar cells, passivating defects in the bulk or at the surface. However, a number of research groups have recently identified hydrogen as being involved with the degradation of multi-crystalline silicon solar cells [1-5]. It is therefore more desirable than ever to have better methods to observe the behavior of hydrogen present in silicon, and how cell processing affects hydrogen transport.

Hydrogen has been recently reported to be involved in an increase in contact resistance between the n-type emitter and screen printed silver contacts, in p-type multi-crystalline silicon solar cells. This has been observed during processes intended to mitigate subsequent degradation associated with hydrogen $[6,7]$. This paper will explore the use of this effect to qualitatively examine hydrogen in crystalline silicon solar cells, and observe its response to varying process conditions. It will be shown that despite the relative simplicity of the experimental apparatus, this approach is highly responsive and capable of providing valuable information on the bound forms of hydrogen in silicon and its transport in interstitial form. In particular, it will be examined how this behavior differs for hydrogen in mono- and multi-crystalline silicon, and in PERC and Al-BSF solar cells.

\section{CONTACT RESISTANCE AND HYDROGEN}

There are two main questions that need to be addressed before the observed changes in series resistance $\left(R_{S}\right)$ can be considered an appropriate technique for studying hydrogen behavior in solar cells; Firstly, can all changes in dark I-V measurements during the process be attributed to changes in contact resistance? Secondly, are these changes due to hydrogen?

The first question has been addressed in previous studies [6, 8 , 9] which use transmission line measurements to correlate $\Delta \mathrm{R}_{\mathrm{S}}$ with a marked increase in contact resistance between the n-type emitter and the silver fingers. Furthermore, observation of the dark IV curves throughout the process at elevated temperatures reveals that the changes are dominated by $R_{S}$.

The question of whether the changes are due to hydrogen is more complex. As with so many effects in silicon for which hydrogen is thought responsible its involvement cannot be directly observed. It can only be inferred. Furthermore, it is not clear by what mechanism hydrogen interacts with the screenprinted contact to increase $\mathrm{R}_{\mathrm{S}}$. However, there are several pieces of evidence that point towards hydrogen being responsible. The first of these is the response to an applied electric field during annealing, which can either enhance or suppress $\Delta \mathrm{R}_{\mathrm{S}}$ [9]. The second is that the $\Delta \mathrm{R}_{\mathrm{S}}$ is highly reversible $[6,9]$. It can be temporarily reversed at room temperature by the application of a forward current in to the device in the dark. It can also be reversed at elevated temperature by changing the applied bias, as further demonstrated in Section III.C.

The nature of the species involved in this effect is therefore highly likely to be that of a charged particle, mobile at room temperature, which is present in high concentrations in silicon solar cells. These characteristics are commonly associated with hydrogen, and this behavior has also been shown to be consistent with simulations of the transport of hydrogen in silicon solar cells. These simulations predict that during annealing hydrogen will re-distribute from the p-type bulk to the n-type emitter [10]. The results in this paper also agree with this hypothesis, demonstrating a dependence of the contact resistance behavior on the bulk material and cell structure.

\section{EXPERIMENTAL METHOD}

The measurement technique used in this work is described in detail in Ref [9]. Fig. 1 presents a schematic of the apparatus used, consisting of 4 probes and a heated stage. $2 \times 1 \mathrm{~cm}$ samples were cleaved from completed industrial cells with hydrogen introduced during co-firing of the metal contacts. These samples were placed on the stage and the probes are used to maintain a constant bias during annealing at temperatures 
between $350-450^{\circ} \mathrm{C}$, as well as to take periodic dark $\mathrm{I}-\mathrm{V}$ measurements. The increase in series resistance $\left(\Delta \mathrm{R}_{\text {S.TEMP }}\right)$ can then be extracted from the dark I-V curves.

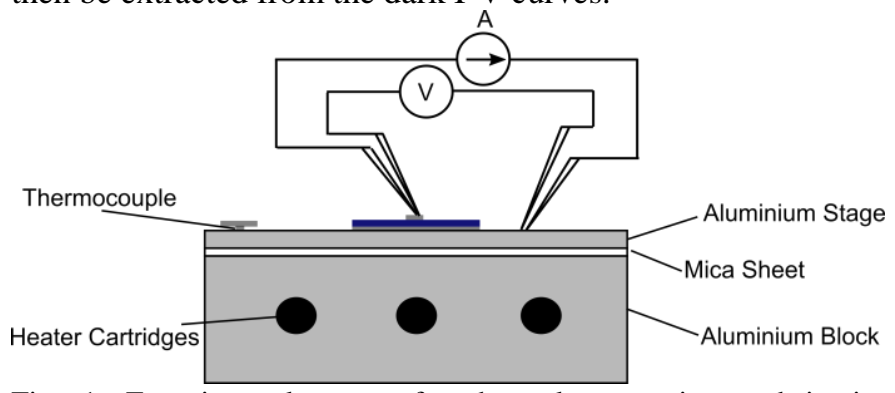

Fig. 1. Experimental set-up for thermal processing and in-situ monitoring of I-V characteristics for solar cell samples [9].

\section{RESULTS AND DISCUSSION}

\section{A. Comparison of Mono- and Multi-Crystalline PERC cells}

Fig. 2 presents the observed change in series resistance at temperatures of 350,400 and $450^{\circ} \mathrm{C}$ to industrial mono- and multi-crystalline PERC solar cells. A $0.2 \mathrm{~V}$ applied bias was applied to reduce the barrier for hydrogen transport to the surface [9]. These results clearly demonstrate a dependence of $\Delta \mathrm{R}_{\text {S.TEMP }}$ upon the bulk material. The rate of increase is lower in multi-crystalline material when compared with monocrystalline and shows a greater temperature dependence.

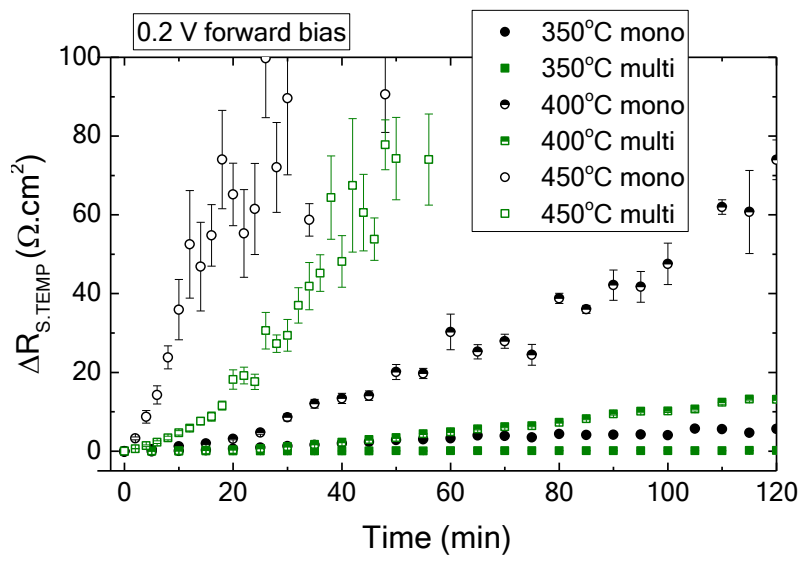

Fig. 2. $\Delta$ Rs.TeMP with annealing time under a $0.2 \mathrm{~V}$ forward bias at temperatures of $300^{\circ} \mathrm{C}$ (filled symbols), $400^{\circ} \mathrm{C}$ (half filled) and $450^{\circ} \mathrm{C}$ (open symbols).

While the motion of hydrogen in silicon is predominantly driven by the interstitial forms $\left(\mathrm{H}^{+}, \mathrm{H}^{0}, \mathrm{H}^{-}\right)$, it is likely that the time-scale of the change in contact resistance is determined by the release of hydrogen from bound forms such as hydrogen dimers and hydrogen decoration of crystallographic defects [11-13]. These results are therefore consistent with the expectation that hydrogen in multi-crystalline silicon, which contains many more impurities and crystallographic defects than mono, is primarily present in bound forms with a higher binding energy.
More information on the bound forms of hydrogen in silicon can be inferred from the behavior of both types of sample under different applied bias as presented in Fig. 3. The internal electric field across the n-p junction will act to oppose transport of $\mathrm{H}^{+}$to the emitter layer [10]. This barrier can be increased by the application of a reverse bias, while a small forward bias is enough to almost completely suppress the built-in potential. This explains why a $0.4 \mathrm{~V}$ forward bias does not significantly increase the rate of series resistance increase compared to a 0.2 $\mathrm{V}$ bias on the multi-crystalline samples. On mono-crystalline samples a larger forward bias is observed to have no observable

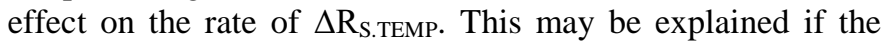
primary form of hydrogen in mono-crystalline silicon is hydrogen dimers, which are reported to have a rate of formation which increases with the electron concentration, which in turn will increase with current [11].
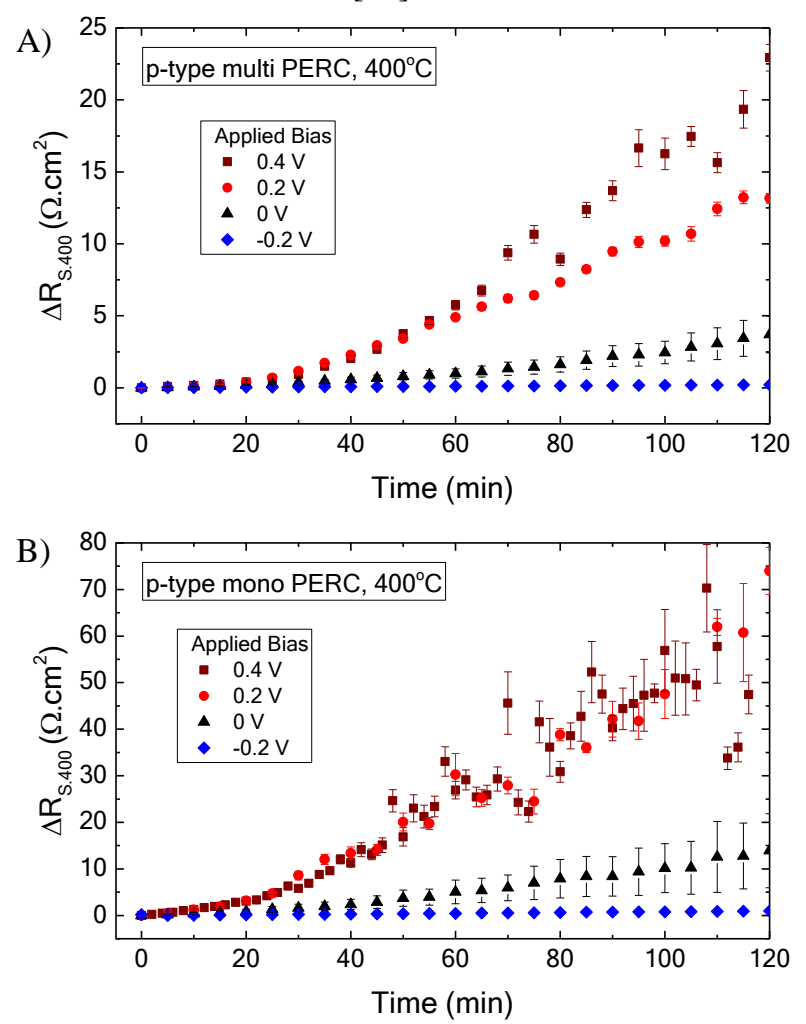

Fig. 3. $\triangle$ Rs.400 for A) multi PERC and B) Mono PERC samples under applied biases from $-0.2 \mathrm{~V}$ to $0.4 \mathrm{~V}$ at $400^{\circ} \mathrm{C}$.

\section{B. Comparison of PERC and Al-BSF Cell Structures}

The hydrogen distribution through the device bulk is expected to be dependent not only upon the bulk material, but upon the device structure. PERC structures are expected to contain more bulk hydrogen than Al-BSF, due to the presence of a second hydrogen source at the rear of the device.

Rate constants for $\Delta R_{S}$ with time were extracted for monoand multi-crystalline industrial Al-BSF and PERC cells under $0.2 \mathrm{~V}$ forward bias. It was not possible to obtain acceptable linear fits to the data however quadratic fits better modelled the behavior, with R-squared values in excess of 0.9 for all 
samples. From these fits the quadratic rate constant, k, was extracted where:

$$
\Delta R_{\text {S.TEMP }}=k t^{2}
$$

Fig. 4 presents an Arrhenius plot of the extracted $k$ for temperatures between $350-450^{\circ} \mathrm{C}$. In the case of monocrystalline samples there is a very clear difference in the rate constant between the two cell structures. Although the apparent activation energies are similar (within the accuracy of these measurements), $k$ for Al-BSF cells are almost two orders of magnitude lower than for PERC cells at each temperature. This is in line with expectations that the Al-BSF structures will contain much less hydrogen. In contrast, in multi-crystalline cells, while at low temperatures $\Delta \mathrm{R}_{\mathrm{S} \text {.TEMP }}$ in PERC cells is much greater, at $400^{\circ} \mathrm{C}$ and above there is no significant difference between the PERC and Al-BSF samples. This is somewhat surprising but could be explained by noting that it is the lower binding energy states in both mono- and multi-crystalline silicon that will be most affected by transport of hydrogen. The higher binding energy sites present in multi-crystalline silicon may well be largely filled by hydrogen regardless of cell structure.

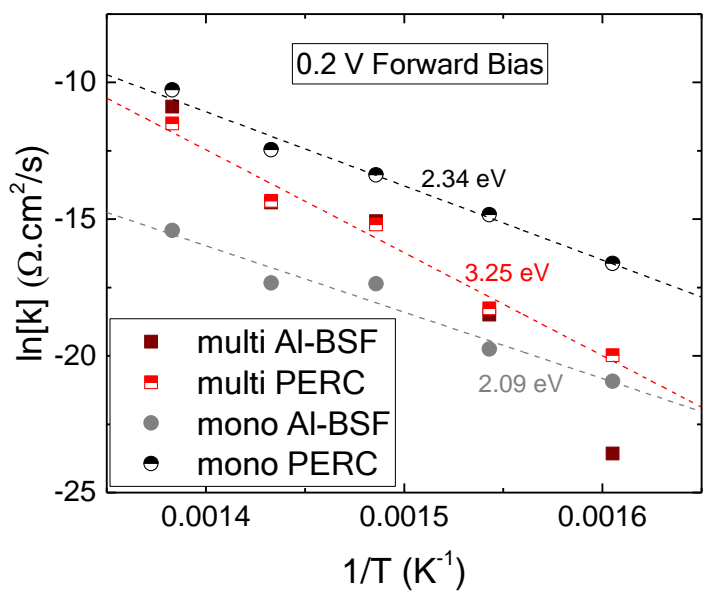

Fig. 4. Arrhenius plot of extracted rate constants (quadratic fits) for cells annealed under a $0.2 \mathrm{~V}$ forward bias at temperatures between $350-450^{\circ} \mathrm{C}$.

\section{Reversibility of Observed Contact Resistance}

The reversibility of the increase in contact resistance at elevated temperatures is an important feature of this effect. Fig. 5 presents $\Delta \mathrm{R}_{\mathrm{S}}$ for mono- and multi-crystalline PERC cells sequentially annealed with forward and reverse biases, compared with samples held at steady voltages. It is readily apparent that not only is the effect highly reversible but the applied biases rapidly switch the samples between two states.

These results provide strong evidence that this effect is hydrogen related, and are in excellent agreement with the expected behavior of hydrogen in silicon. The applied bias (and hence electric fields) affects the highly mobile, interstitial hydrogen and its distribution between the bulk and the emitter of the device, and can be rapidly switched [9]. However, the amount of interstitial hydrogen is determined by the release and re-capture of hydrogen to and from its bound forms. Throughout the anneal the concentration of interstitial hydrogen within the device increases, presumably as more hydrogen is released from its bound forms.
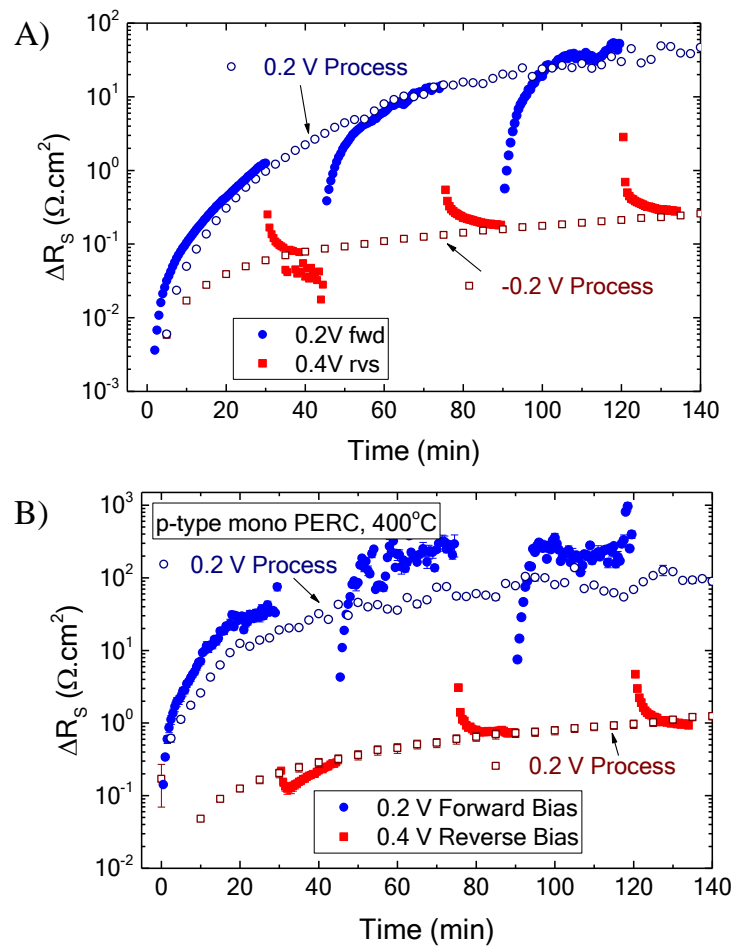

Fig. 5. $\triangle \mathrm{R}_{\mathrm{S}}$ for A) multi PERC and B) mono PERC cells annealed at $400^{\circ} \mathrm{C}$ with an applied bias switched between 0.2 and $-0.4 \mathrm{~V}$. The open symbols represent samples annealed with a fixed bias of $0.2 \mathrm{~V}$ (open blue circles) and $-0.2 \mathrm{~V}$ (open red squares).

\section{Effect on Subsequent Degradation}

It has been previously observed [6] that there is a correlation between the conditions at which the greatest increases in contact resistance take place and those that are most effective at suppressing LeTID in multicrystalline solar cells. Using the application of both forward and reverse biases it is possible to investigate whether this is a causative link, i.e. whether in order to suppress LeTID hydrogen must be re-distributed to the surface and hence to the metal contacts, or not.

$42 \times 1 \mathrm{~cm}$ mono and multicrystalline PERC cell samples were annealed at $400^{\circ} \mathrm{C}$ for 2 hours, 2 with a forward bias of $0.2 \mathrm{~V}$ and 2 with a reverse bias of $0.2 \mathrm{~V}$. These samples were then subjected to light soaking with an illumination intensity of 1 sun at $75^{\circ} \mathrm{C}$. Characterization was carried out at intervals using standard I-V measurements (1 sun, $\left.25^{\circ} \mathrm{C}\right)$, Suns- $\mathrm{V}_{\mathrm{OC}}$ measurements (Sinton WCT-120) [14] and Photoluminescence Imaging (BTi Imaging R1) [15]. The cell parameters as a function of light soaking time are presented in Fig. 6, while the photoluminescence images for selected times are shown in Fig. 7. 


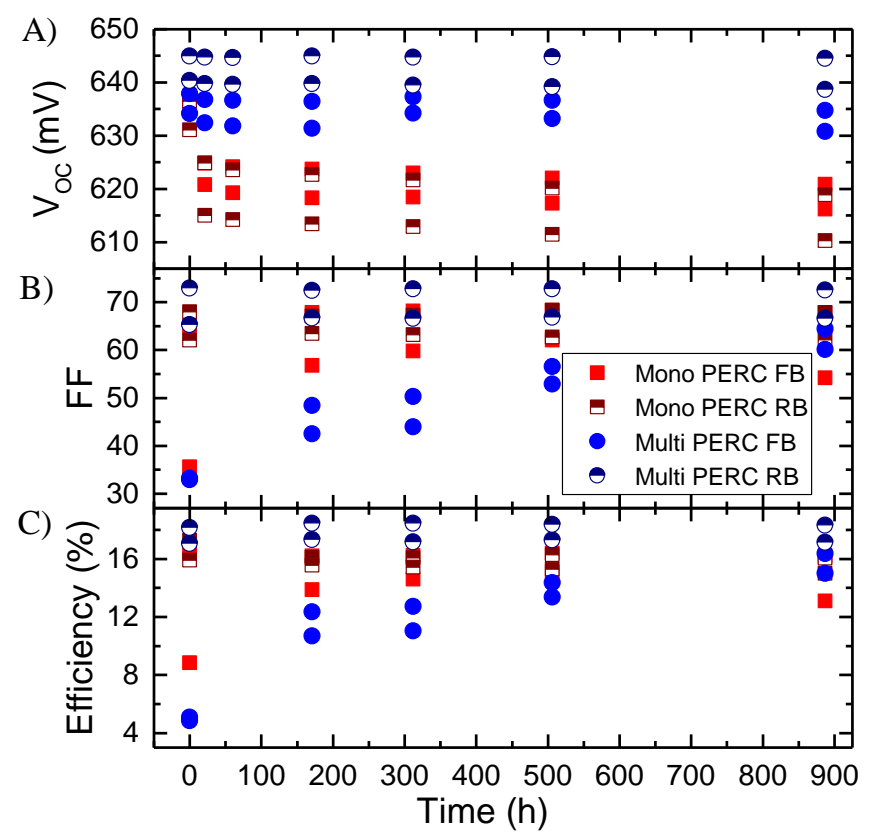

Fig. 6. A) Open-circuit voltage, B) Fill Factor and C) Efficiency of mono- (red squares) and multicrystalline (blue circles) devices measured during light soaking at $70^{\circ} \mathrm{C}$ and 1 sun. Devices were previously annealed at $400^{\circ} \mathrm{C}$ for 2 hours under $0.2 \mathrm{~V}$ forward ( $\mathrm{FB}$, solid symbols) and reverse (RB, half open symbols) bias.

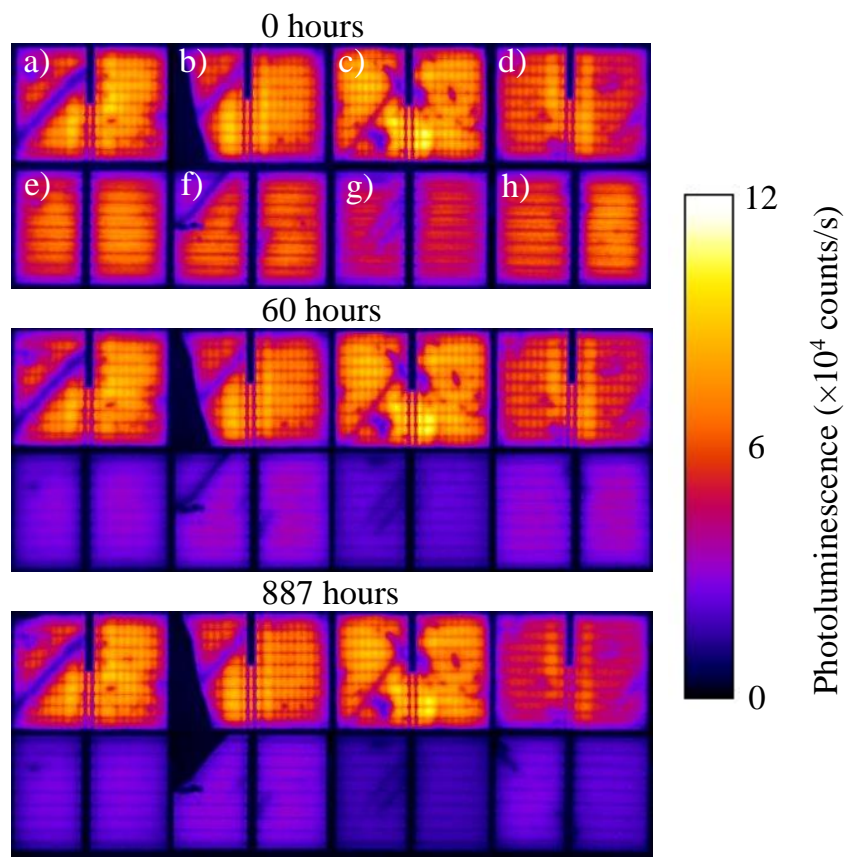

Fig. 7. Photoluminescence images of; a),b) multicrystalline cells annealed under a $0.2 \mathrm{~V}$ forward bias, c),d) multicrystalline cells annealed under of $0.2 \mathrm{~V}$ reverse bias, e),f) monocrystalline cells annealed under a $0.2 \mathrm{~V}$ forward bias, g),h) monocrystalline cells annealed under of $0.2 \mathrm{~V}$ reverse bias. Devices imaged before light soaking, after 60 hours and after 887 hours of light soaking at $70^{\circ} \mathrm{C}$ with 1 sun illumination.
The first point of interest is that the applied bias has had no significant impact upon the subsequent degradation. With both forward and reverse biases applied the multi-cells showed negligible degradation in $\mathrm{V}_{\mathrm{OC}}$ and PL response over more than 800 hours. In contrast the monocrystalline cells $\mathrm{V}_{\mathrm{OC}}$ dropped between $11-16 \mathrm{mV}$ within the first 21 hours, with no subsequent recovery observed.

The applied bias did however have an enormous impact upon the fill factor and ultimate efficiency of the devices. With the exception of one outlier, cells with a forward bias applied had initial fill factors below $40 \%$ upon initial testing, increasing to $45-60 \%$ after 300 hours of light soaking. Samples annealed under a reverse bias also showed reduced fill factors when compared with industrial standard cells, however the effect was much less significant.

These results imply that there is no causative link between the observed increase in contact resistance and subsequent LeTID in multicrystalline silicon solar cells. This means that it should be possible to find a combination of time, temperature and applied bias that would suppress LeTID, without severe degradation of the device fill factor. It may even be possible to apply a very short duration reverse bias at elevated temperature subsequent to such processes in order to largely reverse changes in the series resistance.

Both the elimination of LeTID in multicrystalline cells and the lack of regeneration of the B-O defect in monocrystalline cells may be regarded as indicators of reduced hydrogen availability under light soaking conditions to participate in defect formation/regeneration $[2,5,16,17]$. However, the reversible series resistance of samples is still increasing after 2 hours of annealing, as seen in Fig. 5, which we interpret as a continuing increase in the concentration of interstitial hydrogen during this process. While it is possible this interpretation is incorrect, given the other evidence presented for the role of hydrogen in this paper it is worth exploring.

The main implication of this interpretation is that the $400^{\circ} \mathrm{C}$ anneal does not denude the bulk of hydrogen, as has been suggested previously [10]. This would also somewhat contradict the assertion that slow cooling after firing could lead to a lower hydrogen concentration in the bulk [5]. This in turn would mean that it is not the total hydrogen concentration that is important for defect formation/regeneration but the concentration of hydrogen in a specific form, as has been recently suggested [18].

Based on our measurements we can infer further properties of this form. Firstly, from the kinetics of defect formation/regeneration at low temperatures it is likely that either the binding energy of this form is relatively low, or that it is mobile at these temperatures and able to interact with other defects and precursors. Secondly this form must not be a significant fraction of the total hydrogen concentration, else we would expect rapid release of interstitial hydrogen from this form in the initial stages of the series resistance measurements. Finally this form of hydrogen must not be created during cools from $400^{\circ} \mathrm{C}$, but instead only be present after rapid cools from higher temperatures, otherwise the $400^{\circ} \mathrm{C}$ process should have no effect on subsequent degradation/regeneration. 
It should be stated again that this interpretation hinges upon the assumption that the continued increase in reversible series resistance indicates that there has been no reduction in the interstitial hydrogen present in the device. The best evidence for the involvement of hydrogen is the reversibility of the process, a hallmark of hydrogen involvement, and the dependence upon bulk material and device structure.

In this paper we have interpreted the increase in reversible series resistance as meaning that the interstitial hydrogen concentration has increased due to release from bound forms, but it is also possible that the contacts become more susceptible to the influence of hydrogen with prolonged annealing at temperatures between $350-450^{\circ} \mathrm{C}$. It is also possible that the concentration of hydrogen present in bound forms decreases during this process, however a significant drop in these concentrations should subsequently lead to a drop in the interstitial hydrogen concentration with sufficient annealing time. To date we have not observed evidence of any such drop with over 10 hours of annealing at $400^{\circ} \mathrm{C}$.

\section{CONCLUSION}

This paper has presented how changes in contact resistance of solar cells, during annealing at elevated temperatures under bias conditions, can be explained through the behavior and transport of hydrogen. It has been shown that elevated temperature contact resistance measurements are a highly responsive technique, and that the observed behavior depends upon the material and device structure of the observed sample.

In order to establish greater confidence in the relation between interstitial hydrogen and contact resistance, as well as the contribution of other effects such as glass thickening, a wider range of samples need to be tested. In particular any cells using a hydrogen lean nitride would provide valuable comparison points.

\section{ACKNOWLEDGEMENTS.}

Work at Oxford University has been supported by the U.K. Government through the International and Industrial Engagement Fund and the Supersilicon project (EP/M024911/1), supported by the Engineering and Physical Sciences Research Council (EPSRC). Work at UNSW has been supported by the Australian Government through the Australian Renewable Energy Agency (ARENA) (ARENA 1-A060 and 1SRI001) and the Australian Centre for Advanced Photovoltaics (ACAP), and the Australian Research Council (DE170100620). The views expressed herein are not necessarily the views of the Australian Government, and the Australian Government does not accept responsibility for any information or advice contained herein.

\section{REFERENCES}

[1] D. Bredemeier, D.C. Walter, and J. Schmidt, "Possible Candidates for Impurities in mc-Si Wafers Responsible for Light-
Induced Lifetime Degradation and Regeneration." Solar RRL, 2017: 2(1): 1700159.

[2] C. Chan, T.H. Fung, M. Abbott, D. Payne, A. Wenham, B. Hallam, R. Chen and S. Wenham, "Modulation of Carrier Induced Defect Kinetics in Multi - Crystalline Silicon PERC Cells Through Dark Annealing." Solar RRL, 2017, 1(2): 1600028.

[3] A. Ciesla, "Advanced laser processing for next-generation silicon wafer solar cells," PhD Thesis, 2017, The University of New South Wales.

[4] M. Jensen, A. Morishige, J. Hofstetter, D. Needleman and T. Buonassisi, "Evolution of LeTID Defects in p-Type Multicrystalline Silicon During Degradation and Regeneration." IEEE Journal of Photovoltaics, 2017. 7(4): p. 980-987.

[5] R. Eberle, W. Kwapil, F. Schindler, M. Schubert and S. Glunz, "Impact of the firing temperature profile on light induced degradation of multicrystalline silicon." physica status solidi (RRL)-Rapid Research Letters, 2016. 10(12): p. 861-865.

[6] C. Chan, P. Hamer, G. Bourret-Sicotte, R. Chen, A. Ciesla, B. Hallam, D. Payne, R.S. Bonilla and S. Wenham, "Instability of Increased Contact Resistance in Silicon Solar Cells Following Post - Firing Thermal Processes." Solar RRL, 2017. 1(11): 1700129.

[7] C. Chan, D. Payne, B. Hallam, M. Abbott, T.H. Fung, A. Wenham, B. Tjahjono and S. Wenham, "Rapid stabilization of high-performance multicrystalline p-type silicon PERC cells." IEEE Journal of Photovoltaics, 2016. 6(6): p. 1473-1479.

[8] A. Peral, A. Dastgheib-Shirazi, V. Fano, J.C. Jimeno, G. Hahn and C. del Canizo "Impact of Extended Contact Cofiring on Multicrystalline Silicon Solar Cell Parameters." IEEE Journal of Photovoltaics, 2017. 7(1): p. 91-96.

[9] P. Hamer, C. Chan, R.S. Bonilla, B. Hallam, G. Bourret-Sicotte, K.A. Collett, S. Wenham and P. Wilshaw, "Hydrogen induced contact resistance in PERC solar cells." Solar Energy Materials and Solar Cells, 2018. 184: p. 91-97.

[10] P. Hamer, B. Hallam, R. S. Bonilla, P. P. Altermatt, P. R. Wilshaw, and S. Wenham, "Modelling of hydrogen transport in silicon solar cell structures under equilibrium conditions." Journal of Applied Physics, 2018. 123(4): p. 043108.

[11] V.V. Voronkov and R. Falster, "Formation, dissociation, and diffusion of various hydrogen dimers in silicon." physica status solidi (b), 2017.

[12] S. Pearton, J. Corbett, and T. Shi, "Hydrogen in crystalline semiconductors." Applied Physics A, 1987. 43(3): p. 153-195.

[13] C.G.Van de Walle, "Energies of various configurations of hydrogen in silicon." Physical Review B, 1994. 49(7): p. 4579.

[14] R. Sinton and A. Cuevas. "A quasi-steady-state open-circuit voltage method for solar cell characterization." in Proceedings of the 16th European Photovoltaic Solar Energy Conference. 2000.

[15] T. Trupke, R. Bardos, M. Schubert and W. Warta, "Photoluminescence imaging of silicon wafers." Applied Physics Letters, 2006. 89(4): p. 044107.

[16] S. Wilking, A. Herguth, and G. Hahn, "Influence of hydrogen on the regeneration of boron-oxygen related defects in crystalline silicon." Journal of Applied Physics, 2013. 113(19): p. 194503.

[17] C. Vargas, K. Kim, G. Coletti, D. Payne, C. Chan, S. Wenham and Z. Hameiri, "Carrier-Induced Degradation in Multicrystalline Silicon: Dependence on the Silicon Nitride Passivation Layer and Hydrogen Released During Firing." IEEE Journal of Photovoltaics, 2018. 8(2): p. 413-420.

[18] Fung, T.H., et al., "A four-state kinetic model for the carrierinduced degradation in multicrystalline silicon: Introducing the reservoir state." Solar Energy Materials and Solar Cells, 2018. 184: p. $48-56$. 